\title{
Analyse und Bewertung alpiner Naturgefahren - eine Daueraufgabe im Rahmen des integralen Risikomanagements
}

\section{Hans Kienholz, Bern}

\section{Alpine Naturgefahren}

Aus der Bewegung von Wasser-, Schnee-, Eis-, Erd- und Felsmassen im Bereich der Erdoberfläche können sich Gefahren für Menschen und Güter ergeben. Im Rahmen der folgenden Betrachtungen geht es um die Beurteilung und Vorhersage von möglichen Prozessen, welche Menschen und Güter direkt gefährden und oft innerhalb sehr kurzer Zeit zu Todesopfern, zu Verletzten, zur Zerstörung von Sachwerten, zu Unterbrüchen von Verkehrswegen und Transportlinien sowie zu ökologischen Schäden führen können. Solche Prozesse sind in Gebirgsräumen vor allem Lawinen, Murgänge, Hochwasser, Überschwemmungen sowie Rutsch- und Sturzbewegungen von Erd- und Felsmassen (Fotos 1 und 2). Ausserdem werden auch tiefgründige Sakkungs- und Kriechbewegungen miteinbezogen, die zwar langsam ablaufen, jedoch erhebliche Schäden an Gebäuden und Infrastrukturanlagen verursachen können. Diese Prozesse werden oft unter dem Begriff "gravitative Naturgefahren» zusammengefasst; sie spielen sich zum grössten Teil in geneigtem Gelände und, mit Ausnahme der Überschwemmungen vorwiegend in Gebirgsräumen ab. In der englischen Sprache wurde daher der Begriff «Mountain Hazards» eingeführt, der hier mit "Alpine Naturgefahren» etwas einengend übersetzt wird. "Alpin" muss in diesem Zusammenhang als "Gebirgsräume betreffend» verstanden werden.

\section{Naturgefahren - Naturrisiken}

Bezogen auf ein betrachtetes Gebiet bzw. eine betrachtete Stelle im Gelände ist ein Naturrisiko definiert als Grösse und Wahrscheinlichkeit eines möglichen Schadens durch einen gefährlichen Prozess (eine «Naturgefahr»), der abhängig ist einerseits von Ausmass und (Eintretens-)Wahrscheinlichkeit dieses gefährlichen Prozesses und andererseits vom Wert, der (Präsenz-)Wahrscheinlichkeit (Expositionswahrscheinlichkeit) und der Verletzlichkeit von Objekten an derselben Gefahrenstelle.

Wenn sich der gefährliche Prozess dann effektiv abspielt und sich das Schadenpotential an der Gefahrenstelle befindet, entsteht ein Schaden (Abb. 1).
Integrales Risikomanagement beinhaltet ein operatives Konzept zur Handhabung von Risiken. In einem idealen und rationalen Verfahren werden oder würden zu Beginn und während jeder menschlichen Aktivität nicht nur der erstrebte Nutzen, sondern auch die allfällig inhärenten Risiken in Betracht gezogen. Auch im Zusammenhang mit Naturrisiken sollte mit einer unvoreingenommenen Analyse der möglichen Gefahren und Verletzlichkeiten begonnen werden. Auf der Basis dieser Analysen hat eine Bewertung - in der Regel in Form einer Zuordnung der Gefahren zu Gefahrenstufen - zu erfolgen (Abb. 3).

Dort, wo die Risiken zu gross erscheinen (z.B. gemessen an einer Schutzzielmatrix gemäss Tab. 2), müssen vorbeugende Massnahmen getroffen werden. Diese umfassen Prävention und Vorsorge mit dem generellen Ziel der Verminderung der Verletzlichkeit von Menschen und Sachwerten gegenüber Naturgefahren. Während die Prävention zum Ziel hat, den Schaden durch eine angemessene Raumnutzung zu vermeiden oder diesen mit Schutzmassnahmen abzuwenden, umfasst die Vorsorge vor allem Handlungen, die helfen sollen, eine Katastrophe zu bewältigen (Bereitstellen und Ausbildung von Rettungskräften, Planung von Einsätzen, Abschluss von Versicherungen).

Im Ereignisfall gilt es zunächst, durch rasche Alarmierung, Rettung und Betreuung der Opfer die Folgen so weit als möglich einzugrenzen und mit Sofortmassnahmen (z.B. Evakuationen, behelfsmässige Barrikaden gegen Hochwasser) die Schäden zu begrenzen. Möglichst rasch müssen wichtige Infrastrukturen instand gestellt werden. Im Hinblick auf die laufend anzustrebende Verbesserung des gesamten Risikomanagements (lessons to learn) müssen ausserdem die Ereignisse und die Interventionen gut dokumentiert werden.

In der Phase der Regeneration schliesslich sind die provisorischen Lösungen in definitive Lösungen überzuführen. Dazu gehören nicht nur der Wiederaufbau und die aktive Überführung des Lebens in den Alltagsbetrieb. Es gilt auch, die Ereignisse und die Einsätze zu analysieren und die nötigen Lehren für alle im Risiko-Managements-Kreis (Abb. 2) erwähnten Akteure zu ziehen. Die in Abb. 2 dargestellten Sektoren Beurteilung und Vorbeugung werden damit sozusagen auf ein höheres Qualitätsniveau angehoben und damit auch bessere Voraussetzungen für die Bewältigung und Regeneration bei einem künftigen Ereignis geschaffen. 


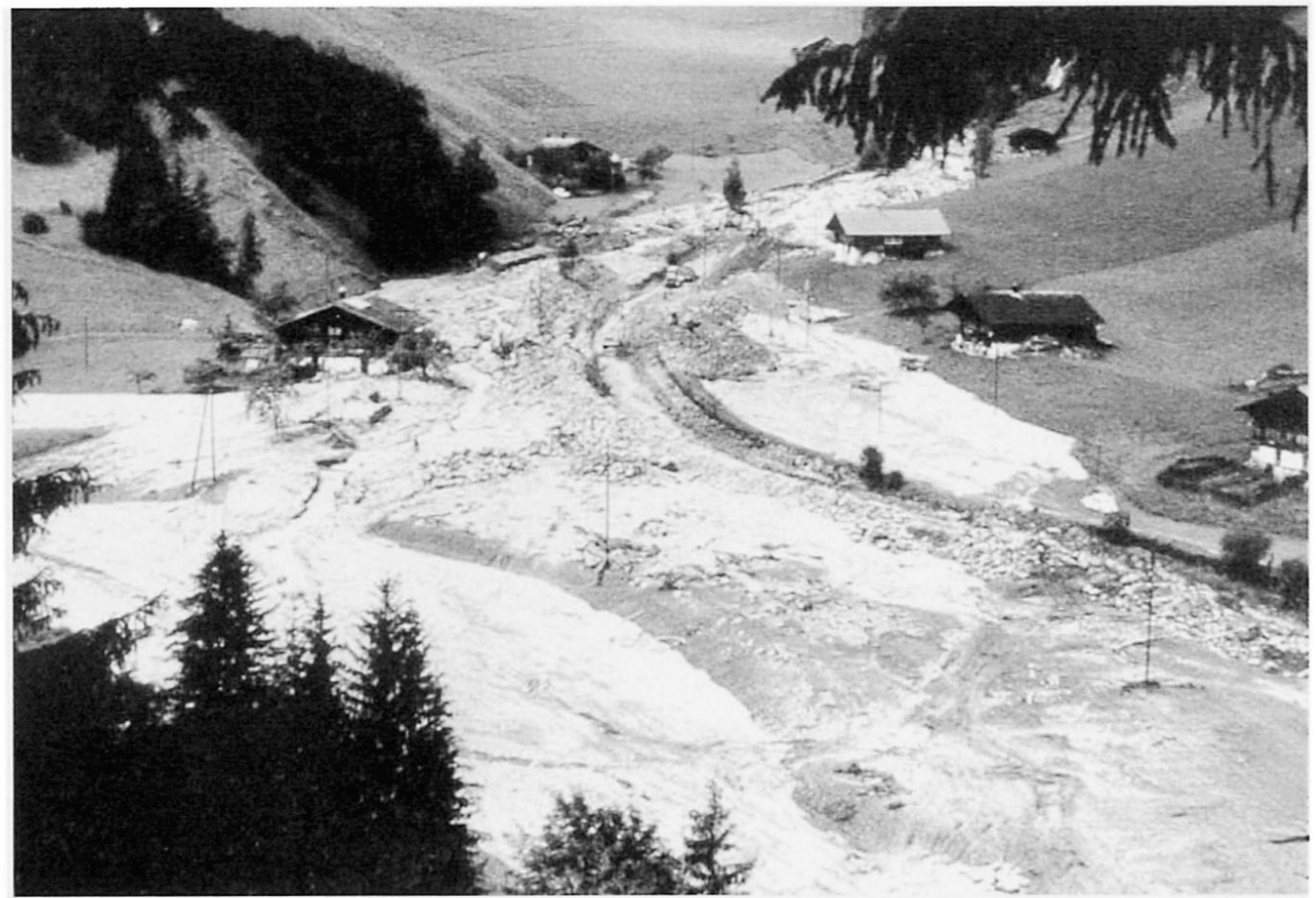

Foto 1: Schadenereignis durch einen Wildbach (Diemtigtal, BE, 1977)

Foto: H. KIENHOLZ Damage caused by a mountain torrent (Diemtigtal, BE, 1977)

Dommage causé par un torrent (Diemtigtal, BE, 1977)

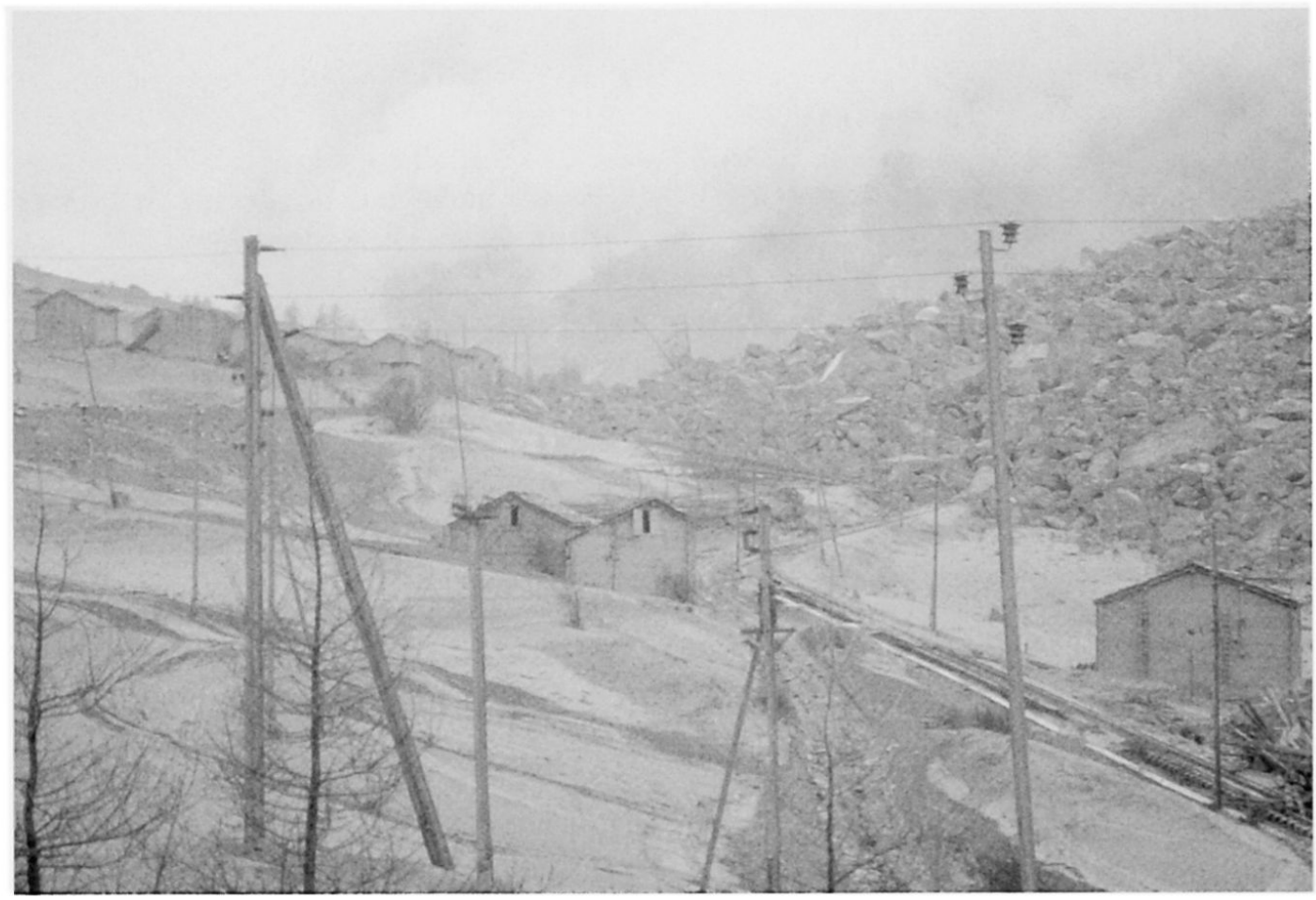

Foto 2: Bergsturz (Randa, VS, 11.5.1991)

Foto: H. KIENHOLZ

Rock avalanche (Randa, VS, 11.5.1991)

Avalanche de rochers (Randa, VS, 11.5.1991) 


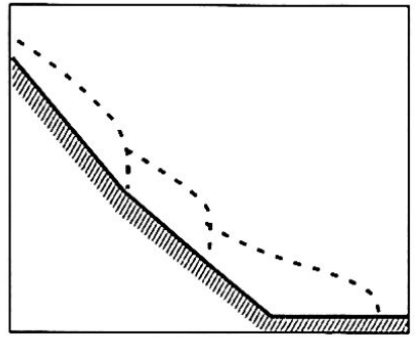

Potentieller gefährlicher Prozess

$=\mathrm{f}$ (Intensität, Wahrscheinlichkeit)

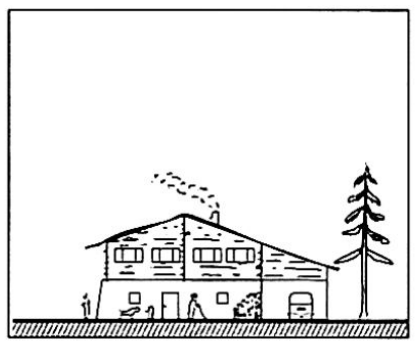

Potentiell

exponierte Werte

$=\mathrm{f}($ Verletzlichkeit, Wert. Expositionswahrscheinlichkeit)

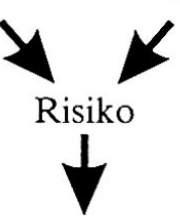

Eingetretenes Ereignis

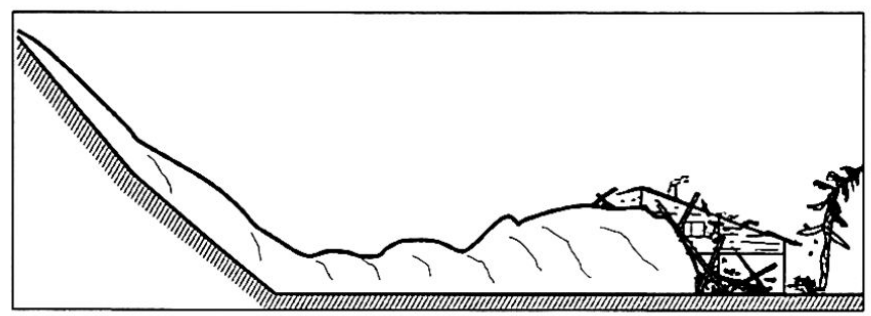

Abb. 1: Gefahr (potentieller gefährlicher Prozess), Schadenpotential (potentiell exponierte Werte), Risiko und Schaden nach eingetretenem Ereignis

Hazard (potentially dangerous process), potential damage (potentially exposed vulnerable elements), risk and damage following events

Danger (processus potentiellement dangereux), dégâts potentiels, risque et dégâts provoqués par un événement

\section{Gefahren- und Risikoanalysen und entsprechende Bewertungen}

Bei jeder Gefahren- und Risikoanalyse und -bewertung ist vorab eine klare Definition des betrachteten Systems (räumliche und zeitliche Abgrenzung, Festlegung der Skalen und der Betrachtungs- und Bearbeitungstiefe) erforderlich.

Ziel der Gefahren- oder der Risikoanalyse ist die Beantwortung der Frage «Was kann passieren?». Dabei werden bei der Gefahrenanalyse nur die gefährlichen (quasi-)natürlichen Prozesse (z.B. Lawine, Murgang) mit ihren grundsätzlichen Wirkungsmöglichkeiten, bei der Analyse des Schadenpotentials zusätzlich die gefährdeten Objekte in ihrem Wirkungsbereich und bei der Risikoanalyse die Schadenwahrscheinlichkeiten und -höhen untersucht (Tab. 1). Die Analysen müssen mit wissenschaftlichen Methoden zu qualitativen und - soweit möglich und sinnvoll - quantitativen, objektiv richtigen Aussagen führen; sie sind dementsprechend klar die Aufgabe von Experten bzw. interdisziplinären Expertenteams. Auch wenn, wie später ausgeführt, die Analyseergebnisse in vielen Fällen mit Unsicherheiten behaftet sind, müssen sie doch so gut abgestützt sein, dass sie als Grundlage für die Gefahren- und Risikobewertung den Stellenwert von Fakten aufweisen.

Ziel der Risikobewertung ist die Beantwortung der Frage «Was darf passieren?» (siehe auch den Beitrag von T. Plattner in diesem Heft). Diese Frage lässt sich nur durch die interessierten bzw. betroffenen stakeholders aufgrund ihrer Risikowahrnehmung und ihrer Abwägung zwischen den Nutzungsmöglichkeiten und den Risiken beantworten. Dabei spielen Lebensumstände, Lebenserfahrung und Wertesysteme eine entscheidende Rolle. Dementsprechend ist Risikobewertung primär Aufgabe jedes Einzelnen (Eigenverantwortung!), aber in vielen Fällen auch klar der Öffentlichkeit, allenfalls delegiert an die gewählten Behörden. Die Risikobewertungen erfolgen somit vor dem Hintergrund des kultur-spezifischen Risikoverständnisses unter Berücksichtigung von ökonomischen und politischen Erwägungen. Die Beantwortung der Frage «was darf passieren?» definiert das akzeptable Risiko. Dabei sind auch indirekte Risiken einzubeziehen, z.B. das Reputationsrisiko eines Touristenortes, in dem sich Unfälle im Zusammenhang mit Naturereignissen häufen.

\section{Zweck und Ausrichtung von Gefahren- und Risikobeurteilungen}

\subsection{Zeitlicher Horizont}

Gefahren- und Risikobeurteilungen im Zusammenhang mit Naturgefahren erfolgen mit verschiedenen Zielsetzungen:

- mit langfristigem Horizont, u.a.:

- Raumplanung (z.B. Siedlung, Anlage von Verkehrswegen)

- Planung von Massnahmen zum Schutz z.B. von (meist schon bestehenden) Siedlungen, Verkehrswegen

- Planung und Vorbereitung von Notfallmassnahmen (inkl. Ausbildung und Training der Einsatzkräfte und regelmässiger Information der Bevölkerung)

- mit kurz- bis mittelfristigem Horizont, u.a.:

- z.B. Evakuationen, Sperrungen von Verkehrswegen zum Zeitpunkt akuter Gefahr

- Regulierung von Gewässern (z.B. Seeabsenkungen bei sich abzeichnender Hochwassergefahr)

- Beurteilung der Lawinengefahr im Hinblick auf 


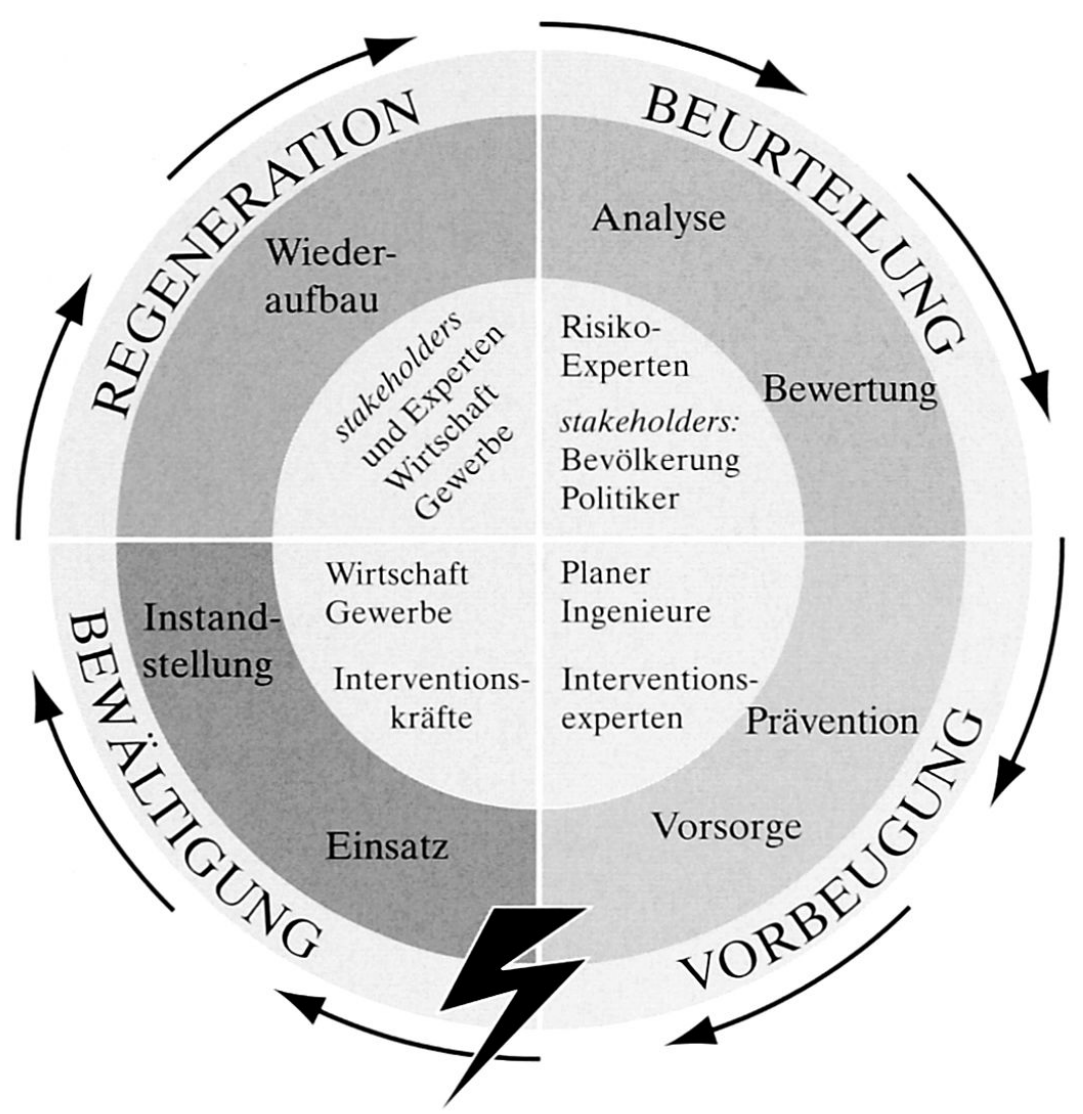

Abb. 2: Der «Risiko-Managements-Zyklus»

Risk management cycle

Le cycle de la gestion du risque

Entwurf: H. Kienholz, Graphik: L. BaumanN

Lawinenwarnungen (durch entsprechende Fachdienste) oder Gefahrenbeurteilung durch den Skitourenfahrer selbst.

Die weiteren Ausführungen im Rahmen dieses Beitrages fokussieren auf Beurteilungen (Analysen und Bewertungen) mit langfristigem Horizont.

\subsection{Gefahren- oder Risikobeurteilung?}

Wo genügen Gefahrenanalysen, wo sind Risikoanalysen und -bewertungen sinnvoll?

Im Bereich technischer Risiken (in der Planungsphase z.B. eines Kernkraftwerks oder eines chemischen Industriekomplexes) sind umfassende Gefahren- und Risikoanalysen und -bewertungen unbestrittenermassen notwendig und sinnvoll.

Im Hinblick auf die Raumplanung wird diese Frage dagegen kontrovers diskutiert:

- Für siedlungsmässig bislang ungenutzte Flächen genügt in der Regel eine Gefahrenkarte als Grundlage. Die Ergebnisse der naturwissenschaftlich-tech- nischen Analyse zusammen mit einem Bewertungssystem (Tab. 1, Abb. 3) sind Aussagen über den Gefährdungsgrad der einzelnen Flächen und der Objekte, die allenfalls in diese Flächen zu stehen kommen sollen. Den Raumplanern genügen diese Aussagen in den meisten Fällen, da sie anhand der ausgewiesenen Gefahrenstufen entscheiden können, ob in einem Gebiet gebaut werden darf, ob das Bauen mit gewissen Auflagen möglich ist, oder ob wegen der Naturgefahren ein Bauverbot auszusprechen ist (KIENHOLZ 1999).

- Bei der Planung von Schutzmassnahmen für bestehende Siedlungen und Infrastrukturen, was gerade in den dichter besiedelten Tälern der Alpen, aber auch im Vorland sehr häufig der Fall ist, stellt sich immer wieder die Frage der Priorisierung von Massnahmen. Denn nicht zuletzt die häufigen und z.T. verheerenden Ereignisse der letzten drei bis vier Dekaden haben deutlich gezeigt, dass nicht alle an sich notwendigen oder wünschbaren Massnahmen technisch machbar sind, oder dass sie am finanziellen Aufwand scheitern. Hier spielt es eine wesentliche Rolle, ob mit einer Erstinvestition von beispiels- 


\begin{tabular}{|c|c|c|}
\hline & $\begin{array}{l}\text { Analyse } \\
\text { «Was kann passieren?» }\end{array}$ & $\begin{array}{l}\text { Bewertung } \\
\text { «Was darf passieren?» }\end{array}$ \\
\hline है & $\begin{array}{l}\text { Gefahrenanalyse: } \\
\text { «Welche Naturgefahren gibt es?» } \\
\text { Naturwissenschaftlich-technische Untersuchung } \\
\text { (Art, Wege, Intensitäten, Frequenzen, } \\
\text { Angriffsweisen) der gefährlichen Prozesse und der } \\
\text { Wirksamkeit allfälliger Schutzmassnahmen } \\
\text { Durchführung durch Expertenteams }\end{array}$ & $\begin{array}{l}\text { Gefahrenbewertung: } \\
\text { «Wie sind die Gefahren einzuordnen?» } \\
\text { Einordnung der Analysenergebnisse in ein } \\
\text { Gefahrenbewertungssystem (z.B. gemäss Abb. 3) } \\
\text { Durchführung durch Expertenteams }\end{array}$ \\
\hline 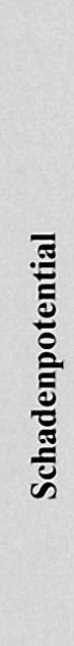 & $\begin{array}{l}\text { Untersuchung (ökonomisch, gesellschaftlich, } \\
\text { ökologisch, technisch) der möglicherweise } \\
\text { exponierten Objekte (Art, materielle und } \\
\text { immaterielle Werte, Verletzlichkeit, } \\
\text { Expositionswahrscheinlichkeit) im möglichen } \\
\text { Wirkungsbereich von Naturgefahren } \\
\text { Durchführung durch Expertenteams }\end{array}$ & $\begin{array}{l}\text { Bewertung des Schadenpotentials: } \\
\text { «Welcher Wert und welche Bedeutung wird dem } \\
\text { Schadenpotential zugemessen?» } \\
\text { Einordnung der Analysenergebnisse in ein (von } \\
\text { der Gesellschaft) explizit oder implizit definiertes } \\
\text { Bewertungssystem (z.B. Schutzzielmatrix } \\
\text { entsprechend Tab. } 2 \text { oder ausgedrückt in } \\
\text { monetären Einheiten); besonders auch Bewertung } \\
\text { von Menschenleben im Vergleich mit Sachwerten } \\
\text { Durchführung durch Expertenteams unter } \\
\text { Berücksichtigung der Wertesysteme der } \\
\text { «Gesellschaft» }\end{array}$ \\
\hline$\frac{0}{3}$ & $\begin{array}{l}\text { Risikoanalyse: } \\
\text { «Mit welcher Wahrscheinlichkeit können durch die } \\
\text { Naturgefahren welche Schäden entstehen?» } \\
\text { Analyse der aus der Koinzidenz von verletzlichen } \\
\text { Objekten und gefährlichen Prozessen } \\
\text { entstehenden Schäden unter Berücksichtigung der } \\
\text { Koinzidenzwahrscheinlichkeit }\end{array}$ & $\begin{array}{l}\text { Risikobewertung: } \\
\text { «Welche Risiken sind akzeptabel, welche nicht?» } \\
\text { Bewertung der Risiken aufgrund der } \\
\text { Risikowahrnehmung (gesteuert durch } \\
\text { Lebenserfahrung, Wertesystem und } \\
\text { Lebensstandard) unter Abwägung des } \\
\text { Verhältnisses zwischen Risiken und Chancen (z.B } \\
\text { Nutzungsmöglichkeiten des betrachteten } \\
\text { Geländes) } \\
\text { Durchführung } \\
\text { Primär individuell (Eigenverantwortung!), } \\
\text { sekundär delegiert an Öffentlichkeit und } \\
\text { Behörden (politische Entscheide) }\end{array}$ \\
\hline
\end{tabular}

Tab. 1: Gefahren- und Risikoanalyse/Gefahren- und Risikobewertung (mit Bezug auf ein räumlich und zeitlich definiertes System) 


\begin{tabular}{|c|c|c|c|c|c|c|}
\hline \multirow{2}{*}{ 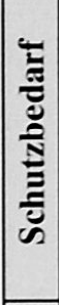 } & \multirow[b]{2}{*}{$\begin{array}{l}\text { Bauten und Mobilien } \\
\text { inkl. Personen in diesen } \\
\text { Bereichen }\end{array}$} & \multicolumn{2}{|l|}{ Objektkategorien } & \multicolumn{3}{|c|}{$\begin{array}{c}\text { Max. zulässige } \\
\text { Intensitätsstufe *) }\end{array}$} \\
\hline & & $\begin{array}{l}\text { Infrastrukturanlagen inkl. } \\
\text { Personen und Mobilien in diesen } \\
\text { Bereichen }\end{array}$ & $\begin{array}{l}\text { Land- und } \\
\text { Forstwirtschaft sowie } \\
\text { ungenutztes Land }\end{array}$ & $\begin{array}{l}\text { 30-jähr } \\
\text { lich } \\
\text { häufig }\end{array}$ & $\begin{array}{l}100- \\
\text { jährlich } \\
\text { selten }\end{array}$ & $\begin{array}{l}\text { 300- } \\
\text { jährlich } \\
\text { sehr } \\
\text { selten }\end{array}$ \\
\hline$\stackrel{\Xi}{\bar{z}}$ & $\begin{array}{l}\text { Standortsgebundene } \\
\text { Bauten, exkl. } \\
\text { Sonderrisiken }\end{array}$ & $\begin{array}{l}\text { z.B. Bergwege, Kletterrouten, } \\
\text { Skitourenrouten }\end{array}$ & $\begin{array}{l}\text { Ödland, } \\
\text { Naturlandschaften }\end{array}$ & 3 & 3 & 3 \\
\hline \multirow{3}{*}{ 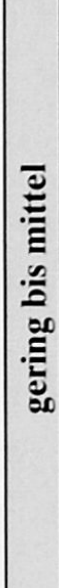 } & & $\begin{array}{l}\text { Wanderwege, Flurwege, } \\
\text { kommunal wichtige Leitungen }\end{array}$ & $\begin{array}{l}\text { Extensiv genutzte } \\
\text { Landwirtschaftsflächen } \\
\text { (z.B. Alpweiden) }\end{array}$ & 2 & 3 & 3 \\
\hline & $\begin{array}{l}\text { Unbewohnte Gebäude } \\
\text { (z.B. Weidescheunen, } \\
\text { Remisen) }\end{array}$ & $\begin{array}{l}\text { Kommunal wichtige Verkehrswege } \\
\text { Regional wichtige Leitungen }\end{array}$ & $\begin{array}{l}\text { Intensiv genutzte } \\
\text { Landwirtschaftsflächen, } \\
\text { Schutzwald }\end{array}$ & 2 & 2 & 3 \\
\hline & $\begin{array}{l}\text { Zeitweise oder dauernd } \\
\text { bewohnte } \\
\text { Einzelgebäude, Weiler, } \\
\text { Ställe }\end{array}$ & $\begin{array}{l}\text { Regional wichtige oder kommunal } \\
\text { sehr wichtige Verkehrswege, } \\
\text { national wichtige Leitungen, } \\
\text { Bergbahnen, Zonen für } \\
\text { Skiabfahrts- und Übungsgelände }\end{array}$ & & 1 & 1 & 2 \\
\hline \multirow{3}{*}{ 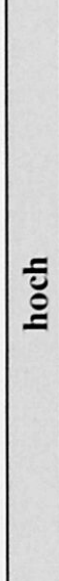 } & & $\begin{array}{l}\text { National wichtige oder regional } \\
\text { sehr wichtige Verkehrswege, Ski- } \\
\text { und Sessellifte }\end{array}$ & & 0 & 1 & 2 \\
\hline & $\begin{array}{l}\text { Geschlossene } \\
\text { Siedlungen, Gewerbe, } \\
\text { Industrie, Bauzonen, } \\
\text { Freizeit-/Sportanlagen }\end{array}$ & $\begin{array}{l}\text { Stationen diverser } \\
\text { Beförderungsmittel }\end{array}$ & & 0 & 0 & 1 \\
\hline & \multicolumn{2}{|c|}{$\begin{array}{l}\text { Sonderobjekte: Objekte mit besonderer Schadenanfälligkeit, } \\
\text { von hohem materiellem oder immateriellem Wert, mit } \\
\text { ausserordentlichen Menschenansammlungen oder mit der } \\
\text { Gefahr von Sekundärschädigungen }\end{array}$} & & \multicolumn{3}{|c|}{ Festlegung fallweise } \\
\hline
\end{tabular}

*) 3 =hoch; 2 = mittel; 1 = gering; 0 = grundsätzlich keine Einwirkung tolerierbar

Tab. 2: Beispiel einer Schutzzielmatrix (Schutzziele = maximal zulässige Intensitätsstufen des «angreifenden» Prozesses unter Berücksichtigung der mittleren Wiederkehrdauer der Ereignisse)

Example of a matrix containing objectives of protection

Exemple d'une matrice des objectifs de protection

Quelle: Bundesamt Für UmWELt, WaLD Und LANDSChaft 1999, I: 34 (verändert)

weise einer Million CHF nur eine einzelne landwirtschaftliche Scheune in der Gemeinde A oder ein dicht überbauter Ortsteil in der Gemeinde B zu schützen ist. Die Risikobeurteilung und darauf aufbauend eine Kosten-Nutzen-Analyse zeigen, dass das Geld im zweiten Falle viel effizienter eingesetzt ist. Aber hier ist zu bedenken, dass mit der Analyse und Bewertung des Schadenpotentials eigentlich nur nachvollzogen bzw. monetarisiert aufgelistet wird, was in den einzelnen Gemeinden an Entwicklung und Expansion in der Vergangenheit stattgefunden hat. Oder, anders ausgedrückt, im Prinzip 


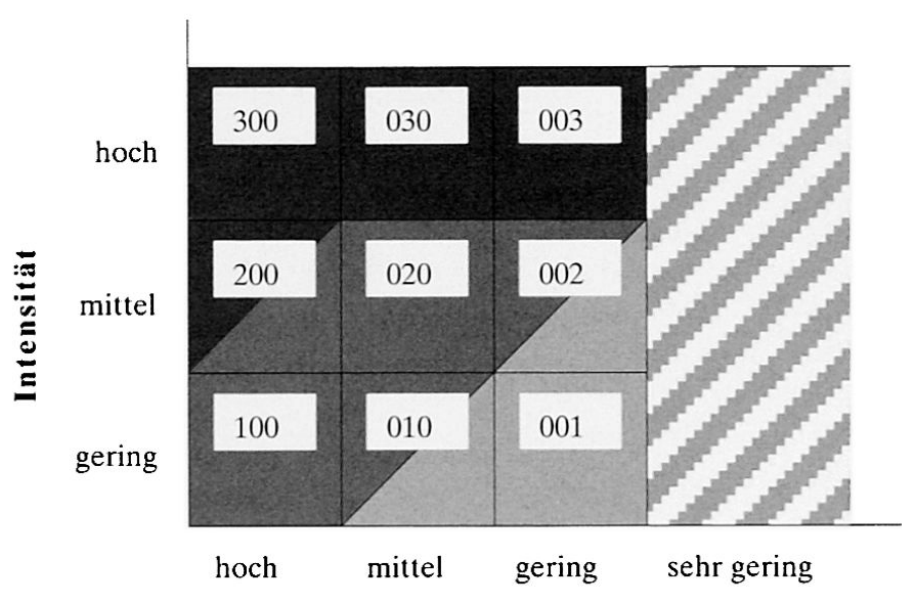

Wahrscheinlichkeit

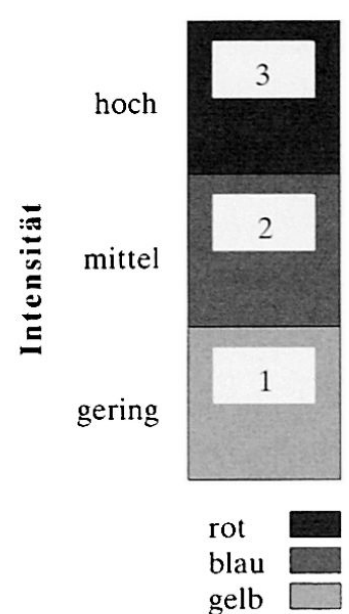

gelb

Abb. 3: Definition der Gefahrenstufen für Gefahrenkarten in der Schweiz (links: Intensitäts-Wahrscheinlichkeits-Matrix für Naturgefahren allgemein, rechts: Intensitätsklassen für permanente Rutschungen, wo die Wahrscheinlichkeit per Definition $=1$ ist)

Definiton of hazard levels in Switzerland (left: general hazards, right: levels of intensity of continuous landslide movements. Probability is defined as 1.)

Définition des niveaux de danger à l'intention de la cartographie des dangers en Suisse (à gauche: matrice de probabilité de l'intensité des dangers naturels en général, à droite: catégories d'intensité en matière de glissements de terrain, pour lesquels la définition/probabilité est égale à 1)

werden bei striktem Vorgehen nach den Ergebnissen der Risikoanalyse die Gemeinde B bzw. die betreffenden Grundbesitzer belohnt, die in der Vergangenheit den Naturgefahren unwissentlich oder auch wissentlich zu wenig Beachtung geschenkt haben; ein entsprechendes Schutzmassnahmenprojekt in der Gemeinde A, die bislang bezüglich Naturgefahren sehr vorsichtig agiert hat, wird nicht realisiert, die Gemeinde wird «bestraft» (dazu auch PetrascheK \& Kienholz 2003: 30).

Diese Überlegungen zeigen in geraffter Form, dass die Diskussion darüber berechtigt ist, wie weit Gefahrenbeurteilungen, wie weit Risikobeurteilungen für die einzelnen Fragestellungen sinnvoll sind. Weiter zeigen sie, dass auch bei klaren, in Zahlenwerten ausdrückbaren Ergebnissen der Risikoanalyse letztlich politische Entscheide zu fällen sind.

Im Folgenden werden nun einige Grundsatzfragen exemplarisch anhand des naturwissenschaftlich-technischen Teils der gesamten Risikobeurteilung, konkret mit Bezug auf die Gefahrenanalyse für die Erarbeitung von Gefahrenkarten erörtert.

\section{Gefahrenanalyse für Gefahrenkarten - Anforderungen}

Bei der Beurteilung von Naturgefahren geht es letzt- lich meist in irgendeiner Form um den Schutz von Menschenleben und Sachwerten vor gefährlichen Naturprozessen. Gefahrenkarten dienen dabei primär dazu, die Raumplanung bezüglich Naturgefahren in vernünftige Bahnen zu lenken und allenfalls Hinweise für notwendige ergänzende Schutzmassnahmen zu geben. Unabhängig von entsprechenden Gesetzen und der Rechtsprechung versteht es sich von selbst, dass Gefahrenbeurteilungen hohen Qualitätsanforderungen genügen müssen. Im Wesentlichen sind

1. sachliche Richtigkeit und

2. gute Nachvollziehbarkeit (Transparenz bezüglich Ablauf der Beurteilung und der eingesetzten Methoden) gefordert.

In der Praxis muss die Beurteilung ausserdem mit angemessenem Zeitaufwand

3. möglichst wirtschaftlich erfolgen.

\subsection{Was heisst «sachlich richtig»?}

So selbstverständlich es ist, mit allen Mitteln eine sachlich richtige Gefahrenbeurteilung anzustreben, so schwierig ist es, im Zusammenhang mit Naturgefahren dieses Ziel zu erreichen und die sachliche Richtigkeit von Gefahrenbeurteilungen zu überprüfen. $\mathrm{Ob}$ eine Gefahrenbeurteilung richtig (gewesen) ist, lässt sich nie absolut ermitteln. Im Prinzip lässt sich erst lange im Nachhinein feststellen, ob sich die gefährlichen Prozesse etwa im Rahmen des Vorhergesagten abgespielt haben oder nicht. 
Grösstmögliche sachliche Richtigkeit wird durch ein Vorgehen nach dem «Stand der Fachkunde» erreicht. Dieser Begriff ersetzt im Kontext des Umganges mit Naturgefahren den Begriff «Regeln der Baukunst» aus dem Bauwesen.

«Es handelt sich um Sachregeln, denen die Rechtsordnung Geltung und damit eine erhöhte Wirksamkeit verleiht.

Der Begriff hat damit eine juristische Tragweite. Konkret sind dies die aktuellen und anerkannten Methoden und Verfahren, wie sie z.B. in der Schweiz von Bundesstellen oder Fachverbänden wie dem SIA empfohlen werden» (Herzog 2000; Kienholz et al. 2002).

Eine Gefahrenbeurteilung entsprechend dem «Stand der Fachkunde» verlangt die Feststellung und Untersuchung aller wesentlichen Aspekte, die zur Gefahr beitragen. Unter anderem gilt es, die verfügbaren Methoden und Prozeduren in sinnvoller Weise nicht nur komplementär, sondern auch bewusst redundant einzusetzen. Im Weiteren müssen beispielsweise auch Summations- und Multiplikationseffekte beim Zusammenspiel verschiedener Naturprozesse oder auch Konflikte zwischen Naturprozessen und Schutzmassnahmen gegen andere Prozesse (z.B. Beeinträchtigung eines Steinschlagschutzwaldes infolge Lawinenschneisen) beachtet werden.

All dies setzt systematisches Vorgehen, aber vor allem auch Aufmerksamkeit in die Tiefe und die Breite, Phantasie (im guten Sinne) und Kreativität des Bearbeiters voraus.

\subsection{Was heisst «nachvollziehbar»?}

Das Vorgehen bei der Gefahrenbeurteilung muss transparent, überprüfbar und nachvollziehbar sein. Nicht zuletzt, weil die Erfüllung des Postulates nach sachlicher Richtigkeit nur beschränkt feststellbar ist, kommt einer hohen Transparenz und guten Nachvollziehbarkeit des Vorgehens grosse Bedeutung zu. Das Vorgehen, die eingesetzten Verfahren und Methoden, die Interpretation der erhobenen Daten lassen sich dadurch besser kontrollieren und überprüfen.

Bei Gefahrenanalysen und -bewertungen sind deshalb in jedem Falle folgende Grundregeln zu beachten:

- Flächendeckende Dokumentation (kartographische Darstellung) des gesamten relevanten Perimeters (Gefahren-Entstehungsgebiet und Gefahren-Wirkungsgebiet, d.h. z.B. Wildbacheinzugsgebiet und Schwemmkegel)

- Klare Methodenwahl und -kombination und deren Offenlegung

- Klar umrissene Entscheidungskriterien bei der Bewertung

- Deklaration der Aussagen, u.a. mit Angabe der Evidenz (Tab. 3).

- Gute Nachvollziehbarkeit des Verfahrens hilft zudem den Bearbeitern bei der Selbstkontrolle und verbessert die Argumentationsbasis bei der Umsetzung.

\subsection{Was heisst «wirtschaftlich»?}

Die Frage der Wirtschaftlichkeit tangiert im vorliegenden Kontext zwei Aspekte. Der erste und wichtigere ist der Aspekt der langfristigen Wirtschaftlichkeit des Umgangs mit Naturrisiken: Die Ergebnisse einer Gefahren- und Risikobeurteilung haben in jedem Fall wirtschaftliche Konsequenzen. Dabei hat eine unvorsichtige, zu optimistische Beurteilung im Ereignisfall neben menschlichem Leid meist auch einen grösseren Schaden zur Folge als dies bei einer richtigen Beurteilung der Fall gewesen wäre. Umgekehrt löst eine übervorsichtige Gefahrenbeurteilung übermässige Reaktionen aus: zu grosse Bauverbotszonen und Einschränkungen (nicht realisierte Nutzungsmöglichkeiten) und oft auch unnötige oder unnötig aufwendig konzipierte Massnahmen.

Jede Gefahrenbeurteilung hat somit volkswirtschaftliche Konsequenzen. Ziel muss es daher sein, die Gefahren und Risiken möglichst «richtig» zu beurteilen.

Der zweite Aspekt - nicht unabhängig vom ersten betrifft die Wirtschaftlichkeit von Projekten zur Erarbeitung von Gefahrenkarten und allenfalls von Massnahmenkonzepten. Die Gefahrenbeurteilung, das Projekt Gefahrenkarte selbst, kostet Zeit und Geld. In der Logik der Wirtschaft, des heute so vehement geforderten Wettbewerbs, des Auftragswesens, der Rahmenbedingungen der staatlichen Auftraggeber und Treuhänder der Steuergelder besteht eine Tendenz zur Kostenminimierung. Ungeachtet der Tatsache, dass die Güte der Gefahrenbeurteilung langfristig über Millioneninvestitionen oder aber Millionenschäden entscheiden kann, besteht leider oft die Meinung, dass eine Gefahrenbeurteilung, die ja nur Papier und Unannehmlichkeiten verursacht, eigentlich immer zu teuer ist. Das kurzfristige Einsparen von Zeit und einigen Tausend Franken bei der Erstellung des Gefahrengutachtens kann längerfristig zu zusätzlichen Kosten in Millionenhöhe führen. Nicht zuletzt auch wegen dieser Problematik wurden in der Schweiz entsprechende Empfehlungen für das Qualitätsmanagement ausgearbeitet (Nationale Plattform Naturgefahren (PLANAT) 1999), die sich an alle beteiligten Akteure richten.

\section{Gefahrenanalyse für Gefahrenkarten - wichtige Aspekte}

Die Gefahrenanalyse erfordert, dass die verschiedenen Gefahrenprozesse nicht nur je einzeln, sondern auch in ihrer gegenseitigen Beeinflussung analysiert und beurteilt werden. Dies führt letztlich bis zu einer 


\begin{tabular}{|c|c|c|}
\hline $\begin{array}{l}\text { Erwiesener } \\
\text { Prozess: }\end{array}$ & $\begin{array}{l}\text { Prozess, der an der betreffenden Stelle «erwiesenermassen» gewirkt } \\
\text { und bleibende Spuren hinterlassen hat (stumme Zeugen) oder der } \\
\text { sonst (z.B. Aufzeichnungen, Zeugenaussagen) dokumentiert ist. }\end{array}$ & \multirow[b]{2}{*}{$\begin{array}{l}\text { Blick in die Vergangenheit: } \\
\text { «lernen aus frïheren } \\
\text { Ereignissen» }\end{array}$} \\
\hline $\begin{array}{l}\text { Vermuteter } \\
\text { Prozess: }\end{array}$ & $\begin{array}{l}\text { Prozess, der an der betreffenden Stelle nicht «erwiesenermassen» } \\
\text { gewirkt hat; der jedoch beispielsweise aufgrund schwer } \\
\text { interpretierbarer Hinweise im Gelände, aufgrund vager Aussagen, } \\
\text { aufgrund allgemeiner Erfahrung oder aufgrund von } \\
\text { Analogieschlüssen (Vergleich mit vergleichbaren anderen } \\
\text { Gefahrengebieten) gewirkt haben dürfte. }\end{array}$ & \\
\hline $\begin{array}{l}\text { Potentieller } \\
\text { Prozess: }\end{array}$ & $\begin{array}{l}\text { Prozess, der an der betreffenden Stelle nicht gewirkt hat, der jedoch } \\
\text { aufgrund der allgemeinen Konstellation (z.B. Topographie, } \\
\text { Geologie, Hydrologie, Vegetation, Waldzustand, Bauten) eintreten } \\
\text { könnte. }\end{array}$ & $\begin{array}{l}\text { Blick in die Zukunft: } \\
\text { "Überlegungen und } \\
\text { Hinweise auf mögliche } \\
\text { Vorgänge infolge } \\
\text { veränderter } \\
\text { Randbedingungen" }\end{array}$ \\
\hline
\end{tabular}

Tab. 3: Evidenz von Prozessen (gefährliche Prozesse und Teilprozesse) Evidence of hazardous processes

Evidence des processus dangereux (processus dangereux et processus partiels)

Analyse des Gesamtsystems aller Einflussfaktoren und Prozesse, die für die gegebene Örtlichkeit von Belang sind.

Ein wichtiges Mittel dazu ist die Feststellung und Untersuchung der wesentlichen Aspekte, die zur Gefahr beitragen. Dazu gehören z.T. in Analogie zur Gefahrenerkennung im Bauwesen folgende Punkte (vgl. dazu Schneider \& SChlatter 1994):

- Korrekte Abgrenzung des zu untersuchenden Perimeters; Berücksichtigung der Vorgänge in den benachbarten Perimetern

- Erkennen und gedankliches Nachvollziehen und Nachrechnen früher abgelaufener Prozesse; $\rightarrow$ Abstützen auf Erfahrungen, dies auch durch Vergleich der vorliegenden Situation mit Gegebenheiten und Ereignissen in anderen Gebieten

- Systembeurteilung: Wie funktioniert das Gesamtsystem der Gefahrenprozesse und seiner Einflussgrössen? Sind auch andere, unerwartete Entwicklungen möglich? Wie sind sie allenfalls zu behandeln? Welchen Einfluss können Umweltveränderungen (z.B. Klima, Landnutzung, Vegetation, menschliche Eingriffe) haben? Welche Modellkonzepte, welche Berechnungsmodelle stehen zur Verfügung? Welche Ergebnisse liefern sie?

Zur Systembeurteilung gehören im Einzelnen auch: - Materialanalyse: Welche Materialien (Gesteine, Wasser, Schnee, Eis, Bäume) sind in welcher Kombination im Spiel?

- Einflussanalyse: Welche Grössen beeinflussen die
Gefahrenprozesse? Wie beeinflussen sich die Prozesse gegenseitig?

- Energieanalyse: Welche Energien sind im Spiel? Mit welchen Massen und welchen Geschwindigkeiten ist zu rechnen? Wo immer sinnvoll und möglich sind hier Modellrechnungen durchzuführen.

- Chronologisches Vorausdenken

- Schwachstellenanalyse

- Kritische Analyse bestehender Gegenmassnahmen (Verbauungen)

- Ableiten und Definieren der relevanten Gefährdungsbilder

- Wirkungsanalyse

- Nachprüfung all dieser Analysen nach erfolgten Ereignissen.

\subsection{Disposition und Auslösung}

Im Zusammenleben mit unseren Mitmenschen (und uns selber!) unterscheiden wir zwischen mehr oder weniger konstantem «Charakter», täglich oder stündlich schwankenden «Launen» und z.T. typischen Reaktionsmustern bei Störungen.

Dasselbe gilt im Prinzip auch für ein Gefahrensystem. Die Vorbereitungsphase eines gefährlichen Prozesses ist in der Regel gekennzeichnet durch eine bestimmte (evtl.zeitlich variable) Disposition für den gefährlichen Prozess und durch ein auslösendes Ereignis.

- Die Disposition zu gefährlichen gravitativen oder hydrologischen Prozessen in Gebirgsräumen ist die 


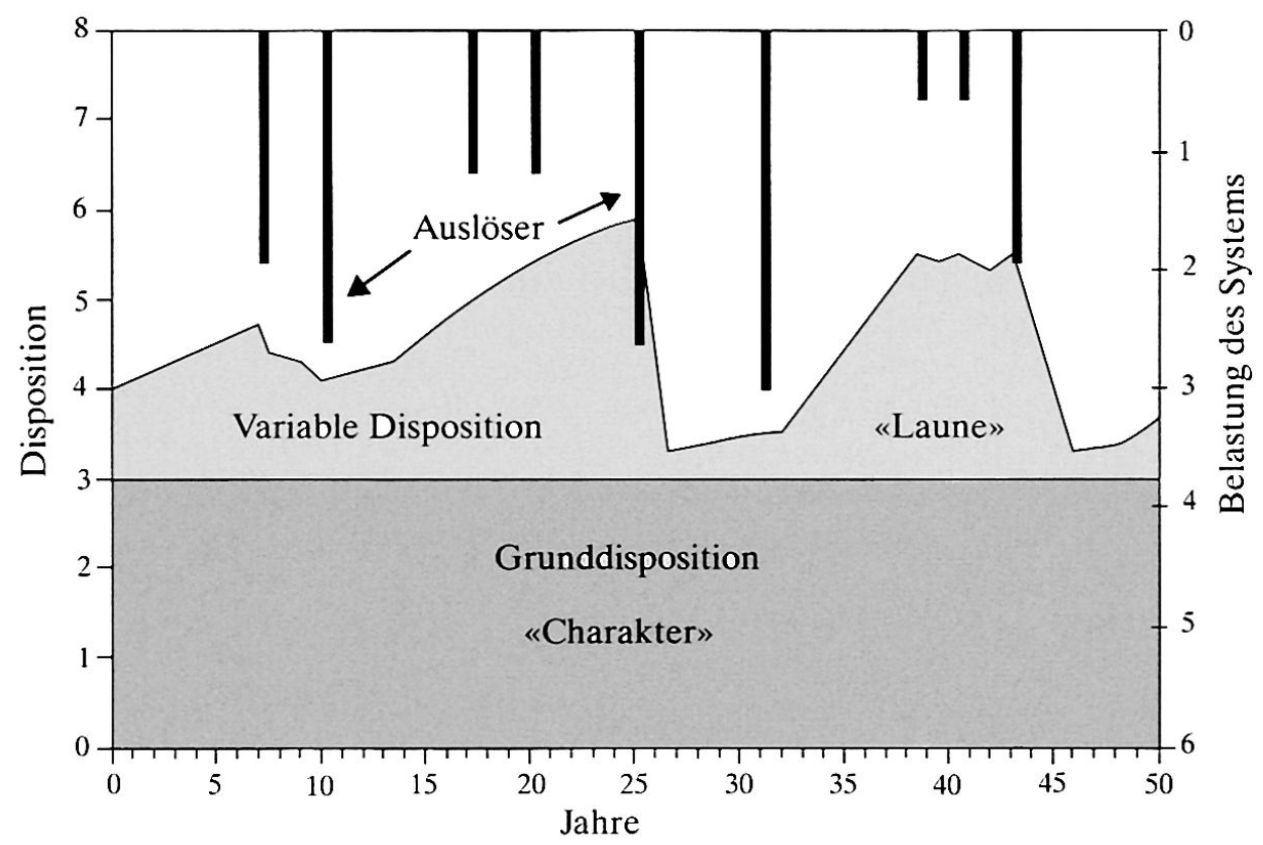

Abb. 4: Disposition und Auslösung von Gefahrenprozessen (beispielsweise Niederschlag als Auslöser der Wildbachaktivität bei gegebener Disposition)

Vulnerability to and triggering of hazardous processes (e.g. thunderstorm can under certain conditions induce increased torrential activity)

Prédisposition et déclenchement de processus dangereux (p. ex. des précipitations orageuses peuvent intensifier l'activité torrentielle)

Entwurf: H. Kienholz, Graphik: L. BaumanN

Anlage oder Bereitschaft von Wasser, Schnee, Eis, Erd- und Felsmassen, sich (in reiner Form oder vermischt) unter dem Einfluss der Schwerkraft so talwärts zu verlagern, dass dies zu Schäden führen kann. Zweckmässigerweise werden dabei eine Grunddisposition und die variable Disposition auseinandergehalten:

- Die Grunddisposition zu gefährlichen Prozessen (der «Charakter») ist die grundsätzliche, über längere Zeit gleichbleibende Anlage oder Bereitschaft zu solchen Prozessen. Die Grunddisposition wird bestimmt durch über längere Zeiträume konstant bleibende Parameter wie Relief, Geologie, Klima, Pflanzenbestand. $\mathrm{Zu}$ beachten ist jedoch, dass einzelne Grössen durchaus einen längerfristigen Entwicklungstrend in eine bestimmte Richtung aufweisen können. So bedeuten das rasche Abschmelzen der Gletscher oder die Hebung der Permafrost-Untergrenze für viele betroffene Gebiete eine Erhöhung der Grunddisposition gegenüber Erosion und Massenbewegungen.

- Die variable Disposition (die «Laune») ist die bei gegebener Grunddisposition zeitlich variable, in einem bestimmten Umfang schwankende oder sich entwickelnde effektive Disposition zu gefähr- lichen Prozessen. Die aktuelle Disposition wird somit bestimmt durch innerhalb eines gegebenen Systemzustandes zeitlich variable, z.T. durch die Jahreszeit und Tageszeit gesteuerte Grössen wie meteorologische Situation, Wasserhaushalt in einem potentiellen Rutschkörper, Vegetationszustand.

- Das auslösende Ereignis, genau genommen jeweils nur das letzte Glied einer Reihe von Gründen (ERISMANN \& ABELE 2001: 109), setzt bei gegebener Disposition den gefährlichen Prozess in Gang (Abb.4).

Diese Unterscheidungen gilt es auch gegenüber gefahrenträchtigen Gesamtsystemen bzw. dem zu beurteilenden Gelände mit allen seinen Aspekten zu machen. Schliesslich können Ausmass und Wahrscheinlichkeit des gefährlichen Prozesses nur dann fundiert abgeschätzt werden, wenn sowohl die Voraussetzungen und Umstände der Vorbereitung als auch die Auslösungsmechanismen der Prozesse erkannt sind.

\subsection{Grundansätze der Gefahrenanalyse}

Eine korrekte Gefahrenbeurteilung erfordert den Einbezug aller verfügbaren Hinweise und Informationen über die gefährlichen Prozesse. Die Verwendung verschiedener methodischer Ansätze und der Vergleich 


\section{Indikationsrichtung}

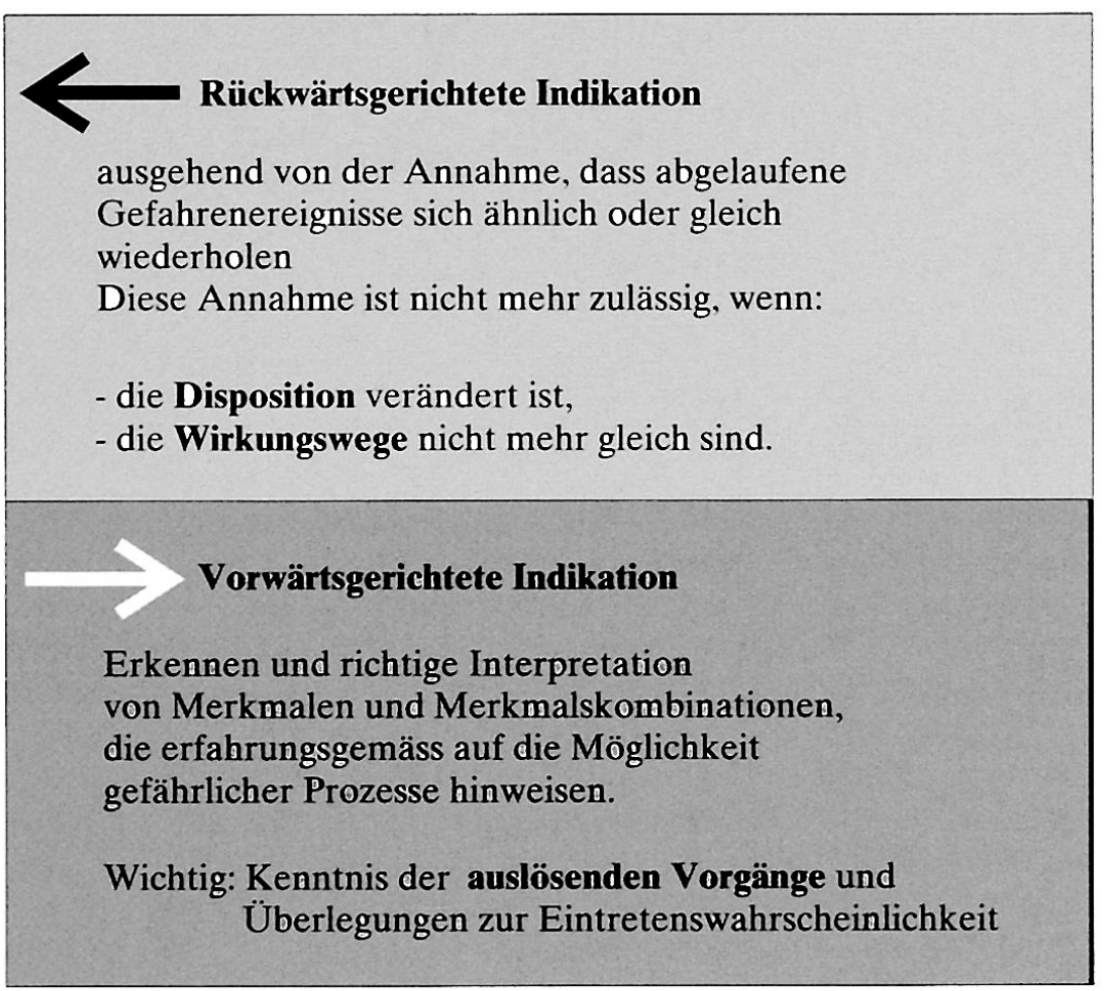

\section{Methodischer \\ Grundansatz}

Auswertung:

von Dokumenten

und Aussagen

zu früheren Ereignissen

\section{Geländeanalyse:}

stumme Zeugen

kritische Konstellationen

und Schlüsselstellen

physische Modelle

mathematische Modelle

Abb. 5: Gefahrenanalyse: Indikationsrichtungen und Grundansätze

Basic methods of hazard analysis

Analyse des dangers: orientations et méthodes de base

mit abgelaufenen Ereignissen (auch in anderen Regionen) schafft die Voraussetzung für die Formulierung realistischer Szenarien.

Die Qualität der Gefahrenbeurteilung wird in hohem Mass durch die verfügbaren Ausgangsdaten bestimmt. Angesichts der oft ungenügenden Datenbasis (vor allem bezüglich quantitativer Daten) ist deshalb jeder Hinweis, jede Information über gefährliche Prozesse zu sammeln und zu interpretieren. Daten und deren Interpretation müssen anhand von Plausibilitätskontrollen, Querkontrollen, Vergleich mit Erfahrungen in anderen Regionen (z.B. Analogien, Anomalien) und weiteren sich anbietenden Möglichkeiten überprüft werden.

Zur Bearbeitung der meisten der oben aufgelisteten Punkte können und müssen verschiedene Ansätze und Verfahren eingesetzt werden (Abb. 5).

Die Auswertung früherer Ereignisse an dem zu beurteilenden Ort (Ereignisanalyse, vgl.Abb. 5) ist in jedem Falle sehr wichtig: Hier erhalten wir Hinweise darüber, was an dem zu beurteilenden Ort alles möglich (gewesen) ist und damit oft unwiderlegbare Argumente zur
Untermauerung der Gefahrenbeurteilung. Im Weiteren liefern sie Kalibrierungsmöglichkeiten von Berechnungsmodellen und nicht zuletzt auch Hinweise zur besseren Abschätzung der Eingangsparameter und deren möglichen Bandbreiten, die dann auch andernorts Anwendung finden können.

In jedem Falle, nicht nur, wenn am betreffenden Ort keine früheren Ereignisse bekannt sind, müssen Gefahrenbeurteilungen prospektiv, vorausschauend durchgeführt werden. Die Randbedingungen können sich inzwischen verändert haben oder neuen Entwicklungen unterliegen. Prospektive Gefahrenindikation erfordert sorgfältige Analysen des Geländes, der Geologie, der Hydrologie und des Gesamtsystems. Eine umfassende Kenntnis der Verfahren, der Modelle, ihrer Stärken, Schwächen, Grenzen, ihrer Empfindlichkeit gegenüber Ausgangsdaten, Annahmen von Randbedingungen ist eine unabdingbare Voraussetzung.

Ebenso grosse Bedeutung haben Szenarienwahl und die Definition der Gefährdungsbilder. Entscheidende Weichenstellungen für die Beurteilung erfolgen in der Szenarienwahl. 
"Werden in dieser Phase Fakten übersehen oder falsch interpretiert, überschatten die Folgen die Detailberechnungen unwiederbringlich» (HeRzoG 2000).

Daraus ergibt sich klar, dass Gefahren- und Risikoanalysen Aufgabe von speziell ausgebildeten und erfahrenen Fachleuten sind, die sich aus verschiedenen Fachdisziplinen rekrutieren können und idealerweise als interdisziplinäre Teams arbeiten.

Grundsätze der Gefahren-Bewertung und die RisikoBewertungen sind dagegen eindeutig Sache der Öffentlichkeit, was nicht ausschliesst, dass sich Fachleute beratend beteiligen. Hier gilt es, Risikokultur bewusst zu leben, d.h. nicht totale Sicherheit um jeden Preis $\mathrm{zu}$ fordern, sondern das notwendige Sicherheitsniveau und die akzeptablen Risiken zu definieren und dann die entsprechenden Massnahmen einzuleiten.

\section{Literatur}

BUNDESAMT FÜR UMWELT, WALD UND LANDSCHAFT (BUWAL) (Hrsg.) (1999): Risikoanalyse bei gravitativen Naturgefahren. - = Umwelt-Materialien Nr.107, Band I: Methode, Band II: Fallbeispiele und Daten, Bern: Bundesamt für Umwelt, Wald und Landschaft.

ErismanN, T.H. \& G. Abele (2001): Dynamics of Rockslides and Rockfalls. - Berlin, Heidelberg, New York: Springer-Verlag.

Herzog, B. (2000): Die Beurteilung von Wassergefahren aus Sicht der neuen Qualitätsempfehlungen. - In: Wasser-Energie-Luft 9-10: 281-285, Baden.

KIENHOLZ, H. (1999): Anmerkungen zur Beurteilung von Naturgefahren in den Alpen. - In: Relief, Boden, Paläoklima 14: 165-184, Berlin, Stuttgart: Gebrüder Borntraeger.

Kienholz, H., Herzog, B., Bischoff, A. \& H.-P. Willi (2002): Fragen der Qualitätssicherung bei der Gefahrenbeurteilung. - Chur: Bündner Wald.

Nationale Plattform Naturgefahren (Planat) (1999): Empfehlungen zur Qualitätssicherung bei der Beurteilung von Naturgefahren. - Biel: Bundesamt für Wasser und Geologie, PLANAT Sekretariat.

Petraschek, A. \& H. Kienholz (2003): Hazard assessment and mapping of mountain risks - example of Switzerland. - In: Rickenmann, D. \& C. Chen: Debris Flow Hazard Mitigation. - Rotterdam: Millpress: $25-38$.

SCHNEIDER, J. \& H.P. Schlatter (1994): Sicherheit und Zuverlässigkeit im Bauwesen. - Zürich: vdf Hochschulverlag AG an der ETH Zürich; Stuttgart: B.G. Teubner Verlag.

\section{Weiterführende Literatur}

Für weiterführende Literatur sei verwiesen auf die Website www.naturgefahren.ch, die regelmässig aktualisiert wird.

\section{Zusammenfassung: Analyse und Bewertung alpiner Naturgefahren - eine Daueraufgabe im Rahmen des integralen Risikomanagements}

Integrales Risikomanagement gegenüber Naturgefahren bzw. Gefahren allgemein bedeutet ein systematisches Vorgehen in einem Kreislauf von Vorbeugung, Bewältigung und Regeneration. Voraussetzung dazu sind vorangehende und begleitende Gefahren- und Risikobeurteilungen.

Gefahren- oder Risikoanalysen dienen der Beantwortung der Frage «Was kann passieren?». Dabei werden bei der Gefahrenanalyse nur die gefährlichen Prozesse (z.B. Lawine, Murgang) mit ihren grundsätzlichen Wirkungsmöglichkeiten, bei der Risikoanalyse aber auch die exponierten Werte und ihre Verletzlichkeit, somit die Schadenwahrscheinlichkeiten und -höhen untersucht.

Zur Beantwortung der Frage «Was darf passieren?» müssen die Risiken bewertet werden. Dies ist eine Aufgabe der Betroffenen, der Öffentlichkeit und geschieht aufgrund der Risikowahrnehmung, der Lebensumstände, der Lebenserfahrung und der Wertesysteme sowie der Abwägung zwischen den Nutzungsmöglichkeiten und den Risiken.

Damit diese hohen Anforderungen mit ihren weitreichenden Konsequenzen erreicht werden können, gilt es, wichtige Grundsätze zur entsprechenden Methodik zu beachten. Einige mögliche Aspekte werden hier angesprochen und begründet.

\section{Summary: Analysis and Evaluation of Natural Alpine Hazards - a continuous process in integral risk management}

With regard to natural and hazards in general, integral risk management is defined as a well organized procedure within a cycle of measures in the areas of prevention, mitigation, coping strategies and regeneration. This presupposes ongoing hazard risk evaluation.

These analyses and evaluations aim to answer the question «what can happen?». However, they are limited to assessment and impact potential of hazardous processes (e.g. snow avalanches, debris flows). This includes the analysis and evaluation of exposed areas and their level of vulnerability, thereby allowing conclusions to be made with regards probability and magnitude of potential damage.

In order to answer questions with regard to permissible levels of damage, potential risks must be evaluated. This is the responsibility of stakeholders and the public, and should weigh chances and risks against each other, as well as include the following criteria: risk perception, personal circumstances, experience and value systems. 
In order to achieve the high demands made on hazard and risk analysis and evaluation, whilst taking the broad scope of consequences into consideration, it is important to pay attention to key principles of appropriate methodologies. The article concludes with a discussion of certain aspects in this connection.

\section{Résumé: Analyse et évaluation des dangers naturels alpins - Une tâche permanente dans le cadre de la gestion intégrale du risque}

La gestion intégrale des risques, par rapport aux dangers naturels et aux dangers en général, constitue une démarche systématique dans le cadre d'un cycle de prévention, de maîtrise et de régénération. Des appréciations tant préalables que simultanées des risques et des dangers sont cependant indispensables.

Les analyses des dangers ou des risques servent à répondre à la question «que peut-il se passer?». L'analyse des dangers ne touche que les processus dangereux (p. ex. avalanche, lave torrentielle) avec leurs impacts possibles. L'analyse des risques permet d'examiner aussi les valeurs exposées et leurs vulnérabilités, donc la probabilité et l'ampleur des dégâts.
Pour répondre à la question «quels événements peut-on tolérer?», les risques doivent être évalués. Ceci est du domaine des personnes concernées et du public, qui apprécieront selon la perception des risques, les conditions de vie, l'expérience et les systèmes de valeur, ainsi que par une évaluation des chances (possibilités d'application) et des risques.

Afin d'atteindre ces exigences élevées aux conséquences à longue portée, il s'agit de respecter les principes fondamentaux de la méthodologie spécifique. Quelques aspects possibles sont ensuite discutés et défendus.

Prof. Dr. Hans Kienholz, Geographisches Institut, Universität Bern, Hallerstrasse 12, CH-3012 Bern. e-mail:kienholz@giub.unibe.ch

\section{Manuskripteingang/received/manuscrit entré le} 12.11.2004

Annahme zum Druck/accepted for publication/accepté pour l'impression: 8.3 .2005 